\title{
Patterns Formed by Droplet Evaporation from a Restricted Geometry
}

\author{
Zhiqun Lin ${ }^{\dagger}$ and Steve Granick* \\ Departments of Materials Science and Engineering, Chemistry, and Physics, \\ University of Illinois at Urbana-Champaign, Urbana, Illinois 61801
}

Received August 29, 2004; E-mail: zqlin@iastate.edu; sgranick@uiuc.edu

The spontaneous emergence of patterns at surfaces is a recurring theme in modern chemistry. Here we are concerned with patterns formed from solvent evaporation, leaving nonvolatile solute left behind. Work in this field has been dominated by the quest for definitive theoretical physical interpretation. ${ }^{1-7}$ Too little attention has been given to the synthesis of patterns of high fidelity and regularity. Here we report a new method to achieve hundreds of concentric rings with definite spacing; each ring is approximately nanometers high and micrometers wide. This simple yet novel approach enables one to produce and organize surface patterns in a well-ordered gradient fashion. The key improvement over past procedures is that droplet evaporation is guided through use of a restricted geometry (Figure 1a), rather than allowing solvent evaporation from a drop sitting on a single solid surface, as in copious past work. ${ }^{1-14}$ Tentatively, we attribute the reason for improvement to the fact that evaporation is constrained to occur at the droplet edges (Figure 1b) rather than allowed over the entire droplet area as in the traditional approach, in which droplets evaporate from a single surface. The main point is that patterns of remarkably high fidelity and regularity result.

A polymer was selected as the nonvolatile component. The selection of this polymer was motivated by the relevance of conjugated polymers to potential optoelectronic devices ${ }^{15}$ but is not believed to be essential to the results presented below. The linear conjugated polymer, poly[2-methoxy-5-(2-ethylhexyloxy)-1,4-phenylenevinylene], end-capped with dimethyl phenyl (MEH-PPV), was dissolved in toluene at concentration $0.5 \mathrm{mg} / \mathrm{mL}$. The $\mathrm{MEH}-$ PPV employed here had the molecular mass of $50-300 \mathrm{~kg} / \mathrm{mol}$. To maximize homogeneity of surface chemistry and topography, single crystals of muscovite mica (ASTM V-2 grade) were cleaved, coated with a reflective film of silver on the backside for subsequent visualization of the contact spot by optical interferometry, and glued onto a cylindrical quartz mount having a diameter of $\sim 1 \mathrm{~cm}$. These methods are adapted from the surface forces apparatus. ${ }^{16}$ The two mica sheets were then placed about $500 \mu \mathrm{m}$ apart with the cylinders at right angles to one another, and a drop of MEH-PPV solution was inserted (Figure 1a). Subsequently, the two curved mica sheets were brought into contact so that they touched; at the apex the geometry was equivalent to that of a sphere-on-flat. As shown in Figure $1 \mathrm{~b}$, this produced a capillary-held polymer solution (capillary bridge) with a diameter of ca. 6-7 $\mathrm{mm}$, depending on the amount of liquid loaded. Experiments were performed at room temperature inside a sealed chamber containing the hygroscopic chemical, phosphorus pentoxide $\left(\mathrm{P}_{2} \mathrm{O}_{5}\right)$. Due to the wetting characteristics of toluene, a concave capillary bridge resulted.

The evaporation generally took hours to complete. Afterward, the two surfaces were separated and examined by optical microscopy in the transmission mode (OM, Olympus, BX51). Figure 2a illustrates that concentric rings of remarkable regularity formed over

Current address: Department of Materials Science and Engineering, Iowa State University, Ames, IA 50011. a

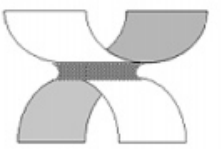

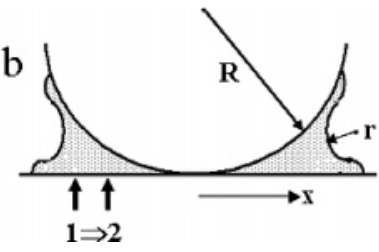

Figure 1. Main idea of this experiment. First (a), a droplet containing solute in volatile solvent is placed in a confined geometry, in this example between crossed cylinders of freshly cleaved single crystals of mica. Next (b), the crossed cylinders are brought together such that a capillary bridge forms with evaporation rate highest at the extremity. This leads to unstable pinning-depinning of the contact line, which moves toward the center of the mica/mica contact as time elapses (denoted in the diagram as $1 \Rightarrow 2$ ) with resulting complex pattern formation, as described in the text.

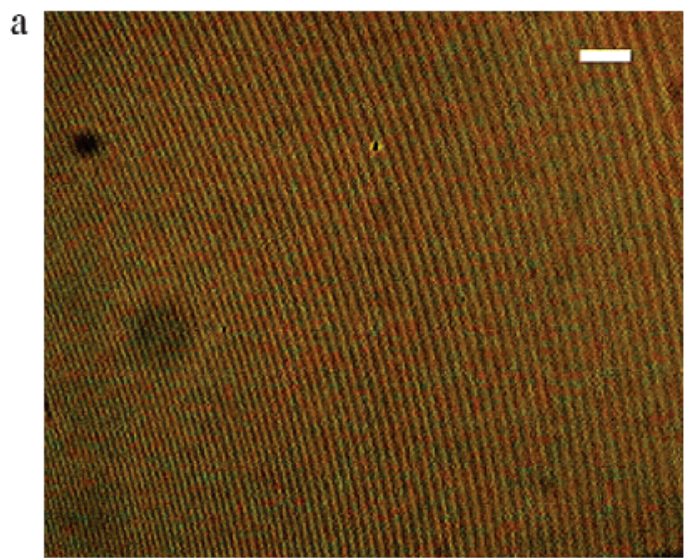

b

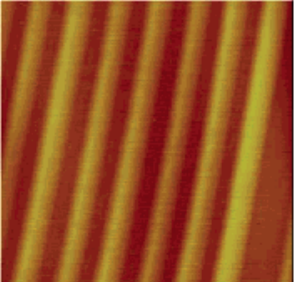

(Position 1)

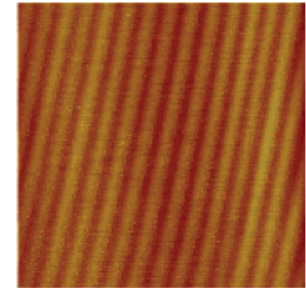

(Position 2)
Figure 2. (a) Optical micrograph of gradient concentric ring patterns formed by deposition of MEH-PPV in the geometry shown in Figure 1b. The white scale bar is $50 \mu \mathrm{m}$, i.e. the linear dimension of the image is $620 \times 528 \mu \mathrm{m}$. (b) AFM images of MEH-PPV stripes as a function of distance as shown in Figure $1 \mathrm{~b}$. The scale is $100 \mu \mathrm{m}$. From the comparison between positions 1 and 2 (the former is positioned farther from the center of the mica/mica contact), one sees that $\lambda_{\mathrm{C}-\mathrm{C}}$ and $h$ depend on radial position.

a lateral distance of several hundred micrometers. (It should be noted that only a small zone of the entire ring pattern is shown in this image.) Qualitatively, it is true that this image resembles Liesegang rings, ${ }^{17,18}$ but it is a false resemblance because the mechanism is fundamentally different. Liesegang rings are produced when diffusion is coupled with chemical reaction, unlike the process that we examine here. ${ }^{17,18}$ It is also worth emphasizing that the size scale of Liesegang rings is typically on the order of millimeters, 


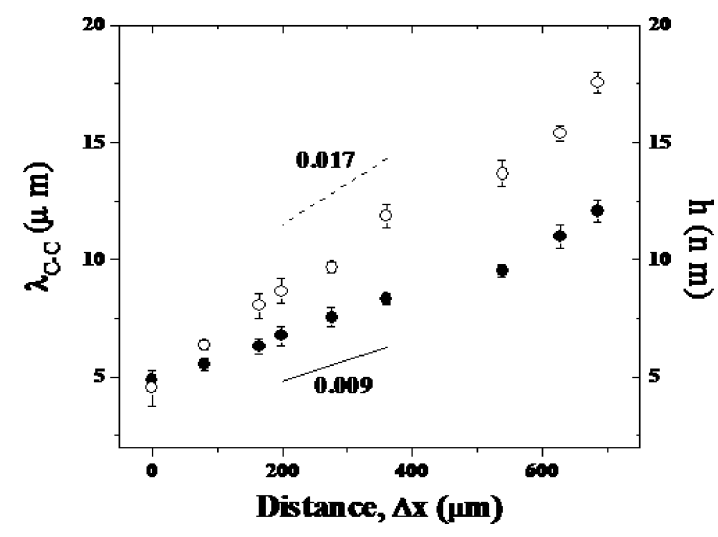

Figure 3. Quantification of data illustrated in Figure 2. $\lambda_{\mathrm{C}-\mathrm{C}}(\bullet)$ and $h$ (O) are plotted as a function of relative distance $(\Delta x)$. Here $\Delta x$ is defined as radial distance relative to $x_{0}=2050 \mu \mathrm{m}$, where $x_{0}$ is from the mica/ mica contact center.

3 orders of magnitude larger than we report here. These patterns also qualitatively resemble those in dry block copolymer films, ${ }^{19}$ but again the resemblance is false because those patterns are produced by the thermodynamics of phase separation between the block copolymer moieties.

To quantify these remarkable patterns, the mica surface was detached from its cylindrical support and imaged using atomic force microscopy (AFM, Digital Instrument, DI 3100). Figure 2b shows representative AFM scans corresponding to positions marked in Figure 1b. The rings seen in Figure $2 \mathrm{a}$ have such a high radius that locally, in the magnified $100 \mu \mathrm{m} \times 100 \mu \mathrm{m}$ scan area in Figure $2 \mathrm{~b}$, they appear as stripes. The distance between adjacent stripes was obtained from fast Fourier transform (FFT) of the AFM image. Figure 3 summarizes the magnitudes of the distance between adjacent stripes $\left(\lambda_{\mathrm{C}-\mathrm{C}}\right)$ and the heights of stripes $(h)$ as a function of distance away from the innermost concentric ring formed in the experiment. One observes that neither $\lambda_{\mathrm{C}-\mathrm{C}}$ nor $h$ was entirely constant; both decreased slowly with increasing proximity to the center of the mica/mica contact, presumably because as the droplet became smaller, the changing radius of curvature of the capillary bridge altered the instability condition between pinning and depinning of the contact line (see below). The relation was not exactly a power law but, to a first approximation, empirically $\lambda_{\mathrm{C}-\mathrm{C}} \sim(\Delta x)^{0.009}$ and $h \sim(\Delta x)^{0.017}$, where $\Delta x$ denotes differential radius. In other words, the pattern was gradient rather than strictly repetitive.

Confirming that the patterns here resulted from having a confined geometry, control experiments were performed in which a drop of $\mathrm{MEH}-\mathrm{PPV}$ toluene solution was placed directly on a single mica surface and allowed to evaporate. Regular concentric ring patterns failed to be formed. It is also noteworthy that the unique ring patterns described here were highly reproducible.

It is easy to extrapolate the ideas presented here to other patterns in which the confining geometry lacks the symmetry of the present study, and also to the use of other materials (nanoparticles, colloids, other polymers). We anticipate that pattern formation will also be predictably governed by parameters that modulate the intensity of surface adsorption, e.g., by chemically modifying the surfaces, the chemical makeup of the nonvolatile component, and the geometrical size of the elementary unit. All these will modulate the tradeoff between stable and unstable contact line pinning on which these patterns appear to depend. It is obvious that this method can be generalized to involve not only capillary bridges held between asymmetric surfaces (which would produce elliptical gradient patterns), but even more interestingly, capillary bridges held between surfaces of tailored wettability (which would produce patterns with broken radial symmetry).
We turn briefly to speculative interpretation. The main point is that, because evaporation in this geometry is restricted to the edge of the drop (Figure 1b), the boundary conditions of "stick" and "slip" of contact with the surfaces surely dominated more than in previous work that involved evaporation from an unconstrained drop. ${ }^{6}$ Symbols in the following discussion are defined in Figure 1b. First, imagine that a contact line formed at an outermost spot of adsorbed MEH-PPV. On the basis of the simple geometrical relation that $x^{2} \approx 4 R r$, it follows that as evaporation proceeded the radius of curvature of the capillary bridge increased. This became unstable and forced the contact line to retract and move to a new stable position to satisfy the imposed relation between $x$ and $r$, at which the contact line again became locked and a second ring of MEH-PPV formed. In our geometry, $R=1.65 \mathrm{~cm}$ and $x=3 \mathrm{~mm}$ initially, so the initial radius of the capillary bridge is $\sim 136 \mu \mathrm{m}$, decreasing below this value as successive rings are formed. The radius and the spacing of the deposited ring patterns must be less than this; their exact values are expected to be also dictated by the rates of the solvent evaporation and solute adsorption at each new contact line as it forms. Therefore, we hypothesize that the observed micrometer-size rings are governed by the imposed geometry, the solution concentration, and the solvent properties. These pinning and depinning processes alternated as toluene evaporated and ultimately, when evaporation was complete, left behind gradient concentric ring patterns uniformly distributed around the droplet center. The use in this study of an exceptionally clean and homogeneous surface enhanced uniformity of these patterns. While it is true that no explanation can be offered at this time of the length scales of periodicity that we observe, we emphasize that, in contrast to the majority of work in this field, which emphasizes the theory of pattern formation, ${ }^{1-7}$ the new preparative strategy described here constitutes a simple and generalizable new approach to prepare periodic gradient features of unprecedented regularity.

Acknowledgment. We are indebted to Alfred Hubler for an illuminating discussion. This work was supported by the U.S. Department of Energy, Division of Materials Science, under Award No. DEFG02-02ER46019 through the Frederick Seitz Materials Research Laboratory at the University of Illinois at UrbanaChampaign. The surface forces equipment was supported under Award No. DEFG02-02ER46019.

\section{References}

(1) Deegan, R. D.; Bakajin, O.; Dupont, T. F.; Huber, G. Nagel, S. R.; Witten, T. A. Nature 1997, 389, 827.

(2) Deegan, R. D. Phys. Rev. E 2000, 61, 475

(3) Deegan, R. D.; Bakajin, O.; Dupont, T. F.; Huber, G. Nagel, S. R.; Witten, T. A. Phys. Rev. E 2000, 62, 756.

(4) Mitov, Z.; Kumacheva, E. Phys. Rev. Lett. 1998, 81, 3427.

(5) Maillard, M.; Motte, L.; Pileni, M. P. Adv. Mater. 2001, 13, 200.

(6) Adachi, E.; Dimitrov, A. S.; Nagayama, K. Langmuir 1995, 11, 1057.

(7) Ohara, P. C.; Gelbart, W. M. Langmuir 1998, 14, 3418.

(8) Okubo, T.; Kimura, K.; Kimura, H. Colloid Polym. Sci. 2002, 280, 1001.

(9) Okubo, T.; Kanayama, S.; Ogawa, H.; Hibino, M.; Kimura, K. Colloid Polym. Sci. 2004, 282, 230.

(10) Kimura, M.; Minser, M. J.; Xu T.; Kim, S. H.; Russell, T. P. Langmuir 2003, 19, 9910.

(11) Nguyen, V. X.; Stebe, K. J. Phys. Rev. Lett. 2002, 88, 164501.

(12) Rabani, E.; Reichman, D.; Geissler, P.; Brus, L. E. Nature 2003, 426, 271.

(13) Xu, S. Q.; Kumacheva, E. J. Am. Chem. Soc. 2002, 124, 1142.

(14) Karthaus, O.; Grasjo, L.; Maruyama, N.; Shimomura, M. Chaos 1999, 9, 308.

(15) Schwartz, B. J. Annu. Rev. Phys. Chem. 2003, 54, 141.

(16) Israelachivili, J. N. Intermolecular and surface forces; Academic Press: London, 1991

(17) Krug, H.; Brandstadter, H. J. Phys. Chem. A 1999, 103, 7811.

(18) Henisch, H. K. J. Cryst. Growth 1986, 76, 279.

(19) Bates, F. S.; Fredrickson, G. H. Phys. Today 1999, 52 (2), 32. JA044792Z 